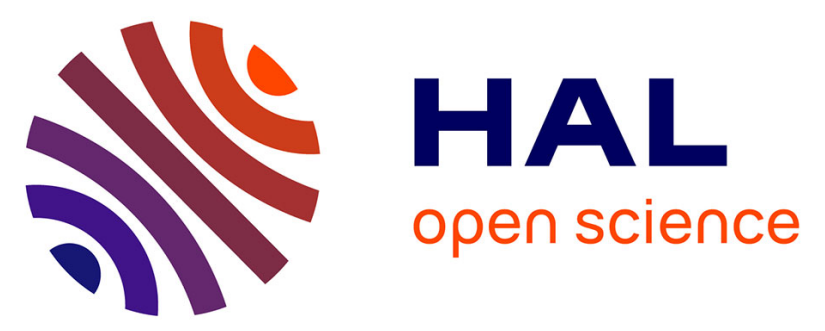

\title{
Dose, image quality and spine modeling assessment of biplanar EOS micro-dose radiographs for the follow-up of in-brace adolescent idiopathic scoliosis patients.
}

\author{
Baptiste Morel, Sonia Moueddeb, Eleonore Blondiaux, Stephen Richard, \\ Manon Bachy, Raphael Vialle, Hubert Ducou Le Pointe
}

\section{To cite this version:}

Baptiste Morel, Sonia Moueddeb, Eleonore Blondiaux, Stephen Richard, Manon Bachy, et al.. Dose, image quality and spine modeling assessment of biplanar EOS micro-dose radiographs for the follow-up of in-brace adolescent idiopathic scoliosis patients.. European Spine Journal, 2018, 27 (5), pp.10821088. 10.1007/s00586-018-5464-9 . hal-02479044

\author{
HAL Id: hal-02479044 \\ https://hal.science/hal-02479044
}

Submitted on 14 Feb 2020

HAL is a multi-disciplinary open access archive for the deposit and dissemination of scientific research documents, whether they are published or not. The documents may come from teaching and research institutions in France or abroad, or from public or private research centers.
L'archive ouverte pluridisciplinaire HAL, est destinée au dépôt et à la diffusion de documents scientifiques de niveau recherche, publiés ou non, émanant des établissements d'enseignement et de recherche français ou étrangers, des laboratoires publics ou privés. 


\title{
Dose, image quality and spine modeling assessment of biplanar EOS micro-dose radiographs for the follow-up of in-brace adolescent idiopathic scoliosis patients
}

\author{
Baptiste Morel ${ }^{1,6}$ (D) Sonia Moueddeb ${ }^{2}$. Eleonore Blondiaux ${ }^{2,3} \cdot$ Stephen Richard $^{2} \cdot$ Manon Bachy $^{4,5}$ (D) \\ Raphael Vialle ${ }^{4,5} \cdot$ Hubert Ducou Le Pointe ${ }^{2,3}$
}

\begin{abstract}
Purpose The aim of this study was to compare the radiation dose, image quality and 3D spine parameter measurements of EOS low-dose and micro-dose protocols for in-brace adolescent idiopathic scoliosis (AIS) patients.

Methods We prospectively included 25 consecutive patients (20 females, 5 males) followed for AIS and undergoing brace treatment. The mean age was 12 years (SD 2 years, range 8-15 years). For each patient, in-brace biplanar EOS radiographs were acquired in a standing position using both the conventional low-dose and micro-dose protocols. Dose area product (DAP) was systematically recorded. Diagnostic image quality was qualitatively assessed by two radiologists for visibility of anatomical structures. The reliability of 3D spine modeling between two operators was quantitatively evaluated for the most clinically relevant 3D radiological parameters using intraclass correlation coefficient (ICC).

Results The mean DAP for the posteroanterior and lateral acquisitions was $300 \pm 134$ and $433 \pm 181 \mathrm{mGy} \mathrm{cm}^{2}$ for the low-dose radiographs, and $41 \pm 19$ and $81 \pm 39 \mathrm{mGy} \mathrm{cm}^{2}$ for micro-dose radiographs. Image quality was lower with the micro-dose protocol. The agreement was "good" to "very good" for all measured clinical parameters when comparing the low-dose and micro-dose protocols (ICC > 0.73).

Conclusion The micro-dose protocol substantially reduced the delivered dose (by a factor of 5-7 compared to the low-dose protocol) in braced children with AIS. Although image quality was reduced, the micro-dose protocol proved to be adapted to radiological follow-up, with adequate image quality and reliable clinical measurements.
\end{abstract}

Keywords Adolescent idiopathic scoliosis · EOS imaging system $\cdot$ Biplanar radiographs $\cdot$ Pediatric radiology $\cdot 3 \mathrm{D}$ measurements 


\section{Introduction}

Imaging plays a major role in the diagnosis and follow-up of adolescent idiopathic scoliosis (AIS). During orthopedic treatment of AIS, adolescents wear a brace and undergo frequent radiological follow-up involving the acquisition of radiographs. The Scoliosis Research Society (SRS) has recently emphasized the clinical relevance and impact of 3D analyses [1,2]. Moreover, the evaluation of spinal deformities in the transverse plane provides new information that is useful in the early stage to evaluate the severity of the scoliosis and impact the therapeutic decisions [3, 4]. EOS low-dose biplanar radiographs (EOS imaging, Paris, France) are particularly adapted to diagnosis and follow-up of AIS, since they allow 3D modeling of the spine in a weight-bearing position, with a significant reduction in radiation dose, compared to computed and digital radiographs [5]. Radiologists must remain vigilant, particularly with a young population undergoing frequent examinations involving ionizing radiations: increased risk of leukemia and brain cancer have been linked to an increase in radiation exposure, even at low doses [6, 7]. This phenomenon has been predicted in pediatric orthopedic patients treated for idiopathic scoliosis, hip dysplasia, and leg-length discrepancy [8]. During spine radiographs, radiosensitive organs such as the thyroid and breasts are exposed to radiation [9]. The cumulative radiation exposure due to periodic full spine radiographs in patients with AIS has recently been estimated to $15 \mathrm{mSv}$, resulting in a significantly increased cancer risk, especially in females $[10,11]$.

According to the "As Low As Reasonably Achievable" (ALARA) principle [12], reducing unnecessary radiation exposure should be a priority, although without compromising the quality of care. A micro-dose protocol is available with the EOS system, which further reduces the radiation dose when compared to the conventional lowdose protocol, and is currently being used in clinical practice $[13,14]$. Assessing the reduction in delivered dose using the micro-dose protocol, and the associated impact on image quality and on the reliability of 3D parameter measurement, is required.

The aim of the present study is to compare the radiation dose, image quality and reliability of 3D spine measurements between the EOS micro-dose and conventional lowdose protocols in braced AIS patients.

\section{Materials and methods}

\section{Study sample, imaging protocol and radiation dose}

Following institutional review board approval, 25 consecutive patients ( 20 females and 5 males) followed for AIS and undergoing brace treatment were prospectively included in this study. The mean age was 12 years (SD 2 years, min 8 years, max 15 years). Exclusion criteria included previous spine surgery and patients with lumbosacral transitional vertebrae (lumbarized S1 or sacralized L5).

For each patient, in-brace biplanar radiographs were acquired with the EOS system (Paris, France) in a standing position, using both the conventional low-dose protocol [15] and the micro-dose protocol $[13,14]$. The acquisition parameters of the micro-dose and conventional low-dose protocols are summarized in Table 1. Radiation exposure, as assessed by the dose area product (DAP), was recorded for the lowdose and micro-dose images (Table 1).

\section{Image quality assessment}

Images were reviewed independently under identical conditions by two experienced radiologists who were blinded to the acquisition protocol. Diagnostic image quality was assessed using a list of 20 anatomical criteria adapted from the "European Guidelines on Quality Criteria for Diagnostic Radiographic Images" [16], which are suitable for a thorough analysis of scoliosis [17]. Nineteen criteria concerned the visibility of anatomical structures (vertebral body, pedicles and spinous process at each spinal level, femoral heads, sacrum, posterior inferior iliac spines, clavicles, chest, mediastinal lines and degree of ossification of the iliac crest, as defined by the Risser index). Each criterion was rated from 1 to 4 , according to the least visible structure for each criterion: (1) structure not detectable, (2) structure visible but features not perceptible, (3) features discernible but not clearly defined, (4) features clearly defined. The 20th criterion was the ability of the observer to measure the Cobb angles reliably and accurately. Both observers were trained

Table 1 Mean $( \pm$ SD) dose area product (DAP) and acquisition parameters ( $\mathrm{kV}$ and $\mathrm{mA}$ ) used during low-dose and micro-dose acquisitions

\begin{tabular}{llllll}
\hline & Low-dose & & & \multicolumn{2}{l}{ Micro-dose } \\
\cline { 2 - 3 } \cline { 5 - 6 } \cline { 5 - 6 } & Posteroanterior & Lateral & & Posteroanterior & Lateral \\
\hline $\begin{array}{llllll}\mathrm{DAP} \\
\left(\mathrm{mGy} \mathrm{cm}{ }^{2}\right)\end{array}$ & $300 \pm 134$ & $433 \pm 181$ & & $41 \pm 19$ & $81 \pm 39$ \\
$\mathrm{kV}$ & 83 & 102 & 60 & 80 \\
$\mathrm{~mA}$ & 200 & 200 & 80 & 80 \\
\hline
\end{tabular}


on a set of images not included in the analysis prior to performing the study.

\section{Statistical analysis}

Statistical analysis was performed with MedCalc software (MedCalc Software bvba, version 12; Ostend, Belgium).

Agreement on image quality assessment between the two radiologists was evaluated using an inter-rater agreement (Kappa, $\kappa$ ) for each of the 20 criteria mentioned above. Agreement was considered "fair" for $\kappa$ values between 0.21 and 0.40 , "moderate" for $\kappa$ values between 0.41 and 0.60 , "substantial" for $\kappa$ values between 0.61 and 0.80 , and "excellent" for $\kappa$ values between 0.81 and 1. A Wilcoxon signed rank test with continuity correction was used to compare the image quality results obtained from the low-dose and micro-dose protocols for the different anatomical structures. Significance was set at $p<0.05$.

Inter-rater reliability of the 3D measurements was assessed with an intraclass correlation coefficient (ICC) and $95 \%$ confidence intervals, for both the low-dose and micro-dose images [12]. An ICC greater than 0.91, between 0.71 and 0.91 , between 0.51 and 0.70 , or less than 0.51 was considered to represent a very good, good, moderate or poor agreement, respectively.

The reliability of micro-dose versus low-dose measurements was then evaluated by utilizing a one-way analysis of variance (ANOVA) comparing the inter-rater measurement differences of both protocols. Significance was set at $p<0.05$.

\section{D modeling and clinical parameters reliability}

Two experienced operators performed the 3D modeling process once for each of the 25 subjects in a random order, for both the low-dose and micro-dose radiographs (total 100 reconstructions). Reconstructions were performed using the "fast spine" workflow with the sterEOS software (EOS imaging, Paris, France). The following clinical measurements were obtained from the 3D models and recorded for each patient: T1T12 and T4T12 kyphosis, L1L5 and L1S1 lordosis, Cobb angle of the main curve, pelvic incidence, pelvic tilt and sacral slope.

\section{Results}

\section{Imaging protocol and radiation dose}

None of the patients required a second acquisition for $3 \mathrm{D}$ analysis (due to excessive motion, image acquisition errors, etc). The micro-dose protocol reduced radiation exposure from factor of 5-7 compared to low-dose [mean DAP was reduced from $300 \pm 134$ to $41 \pm 19 \mathrm{mGy} \mathrm{cm}^{2}$ for the posteroanterior (PA) radiographs, and from $433 \pm 181$ to $81 \pm 39 \mathrm{mGy} \mathrm{cm}^{2}$ for the lateral (LAT) radiographs].

\section{Image quality assessment}

The mean inter-rater agreement $(\kappa)$ based on 100 images (50 PA and 50 LAT) studied with the 20 anatomical criteria defined earlier was 0.94 . The mean results for each of the image quality criteria, between low-dose and micro-dose images, are detailed in Table 2.

\section{Reliability of 3D modeling and clinical parameter measurements}

The anatomical landmarks that are required for the $3 \mathrm{D}$ reconstruction process were visible on all radiographs, and their identification was possible in all cases (Fig. 1). Parameters obtained from the 3D spine models reconstructed from the low-dose and micro-dose stereoradiographs are reported in Table 3. Inter-rater reliability of the 3D measurements was good to very good ( ICC > 0.73). Detailed results for each measurement are reported in Table 4. No statistical difference was found between low-dose and micro-dose measurements, as reported in Table 5.

\section{Discussion}

Radiography plays a major role in confirming the initial diagnosis of AIS and is essential for follow-up and the evaluation of curve progression over time. Orthopedic treatment of AIS with bracing requires regular assessment through medical imaging every 6-24 months, to assess the risks and benefits of different treatment options. For children with AIS, Presicutti et al. have reported that the mean number of radiographs per year was up to 12 for those undergoing surgical treatment, and 5.7 for those undergoing bracing treatment [18]. Decreasing radiation exposure associated with imaging, especially in these populations, remains an important objective for radiologists, because of their potentially increased risk of developing cancer. Low-dose EOS images and 3D models provide a useful tool for the radiological follow-up of AIS [13]. Law et al. have shown that the maximal cumulative effective dose from an early onset patient undergoing semi-annual EOS full spine scan from 10 up to 18 years old was $1.85 \mathrm{mSv}$ for girls and $1.52 \mathrm{mSv}$ for boys, which was about seven times in using EOS system when compared with using digital imaging systems [19]. The micro-dose protocol promises to allow further dose reduction for these at-risk patients. The aim of our study was to compare the radiation dose, image quality and $3 \mathrm{D}$ 
Table 2 Visibility of anatomical structures in posteroanterior (PA) and lateral (LAT) radiographs for both low-dose and micro-dose radiographs

\begin{tabular}{|c|c|c|c|c|c|c|}
\hline & \multicolumn{2}{|c|}{$\begin{array}{l}\text { Visibility of anatomical struc- } \\
\text { tures (score 1-4) } \\
\text { PA view }\end{array}$} & \multirow[t]{2}{*}{$\begin{array}{l}\text { Wilcoxon signed } \\
\text { rank test }(p)\end{array}$} & \multicolumn{2}{|c|}{$\begin{array}{l}\text { Visibility of anatomical struc- } \\
\text { tures (score 1-4) } \\
\text { LAT view }\end{array}$} & \multirow[t]{2}{*}{$\begin{array}{l}\text { Wilcoxon } \\
\text { signed rank } \\
\text { test }(p)\end{array}$} \\
\hline & Low-dose & Micro-dose & & Low-dose & Micro-dose & \\
\hline Cervical vertebral body & 2.7 & 2.3 & $\mathbf{0 . 0 3}$ & 2.6 & 2.5 & 0.13 \\
\hline Cervical pedicle & 2.8 & 2.4 & $\mathbf{0 . 0 3}$ & 2.3 & 2.2 & 0.48 \\
\hline Cervical transverse processes & 2.2 & 1.7 & 0.01 & 1 & 1 & NA \\
\hline Cervical spinous process & 3.2 & 3.1 & 0.41 & 2.5 & 2.4 & 0.39 \\
\hline Thoracic vertebral bodies & 2.9 & 2.5 & 0.005 & 1.5 & 1.3 & 0.07 \\
\hline Thoracic pedicles & 3.1 & 2.9 & 0.06 & 1.3 & 1.1 & 0.16 \\
\hline Thoracic transverse processes & 1.6 & 1.3 & 0.09 & 1 & 1 & NA \\
\hline Thoracic spinous processes & 2.1 & 1.9 & 0.12 & 1 & 1 & NA \\
\hline Clavicles & 4.0 & 3.9 & 0.59 & 1 & 1 & NA \\
\hline Chest & 3.5 & 3.2 & 0.01 & 2.5 & 2.3 & 0.01 \\
\hline Mediastinal lines & 3.6 & 3.3 & 0.02 & 2.4 & 2.2 & $\mathbf{0 . 0 3}$ \\
\hline Cobb & 3.9 & 3.9 & 0.58 & 1 & 1 & NA \\
\hline Lumbar vertebral body & 3.6 & 2.8 & 0.0001 & 3.1 & 2.8 & 0.01 \\
\hline Lumbar pedicles & 3.5 & 3 & 0.0004 & 2.7 & 2.3 & 0.001 \\
\hline Lumbar transverse process & 2.5 & 1.8 & 0.0004 & 1 & 1 & NA \\
\hline Lumbar spinous process & 3.3 & 2.7 & 0.001 & 2.2 & 1.7 & 0.006 \\
\hline Femoral heads & 3.7 & 2.7 & 0.0001 & 2.1 & 1.6 & 0.0008 \\
\hline Posterior inferior iliac spines & 3.5 & 3 & 0.0009 & 1.6 & 1.4 & 0.04 \\
\hline Sacrum & 2.4 & 1.9 & 0.0003 & 3.4 & 2.8 & 0.0002 \\
\hline Risser index & 3.9 & 3.8 & 0.09 & 1 & 1 & NA \\
\hline
\end{tabular}

Scores are presented as the mean between the two radiologists for each criterion. Significant differences are highlighted in bold

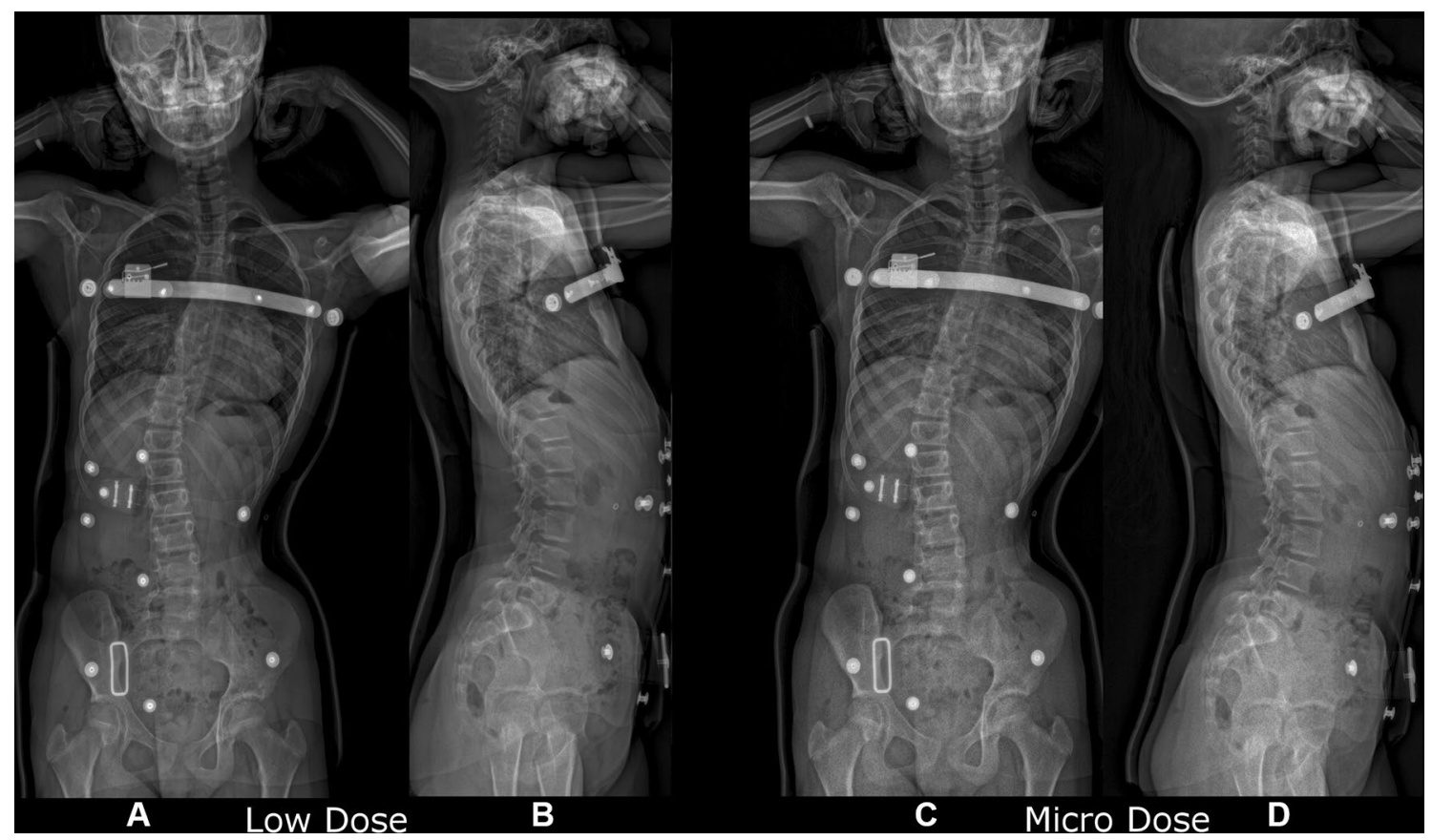

Fig. 1 Comparison of the frontal (PA) and lateral (LAT) views of a AIS patient obtained with conventional low-dose EOS (a, b) and with the micro-dose protocol $(\mathbf{c}, \mathbf{d})$ 
Table 3 Descriptive 3D radiographic measurements (in degrees) obtained using the low-dose $(n=25)$ and micro-dose biplanar stereoradiographs $(n=25)$

\begin{tabular}{|c|c|c|c|c|c|c|c|c|c|}
\hline & & Cobb angle & $\begin{array}{l}\text { T1-T12 } \\
\text { kyphosis }\end{array}$ & $\begin{array}{l}\text { T4-T12 } \\
\text { kyphosis }\end{array}$ & $\begin{array}{l}\text { L1-L5 lor- } \\
\text { dosis }\end{array}$ & $\begin{array}{l}\text { L1-S1 lor- } \\
\text { dosis }\end{array}$ & $\begin{array}{l}\text { Pelvic inci- } \\
\text { dence }\end{array}$ & Sacral slope & Pelvic tilt \\
\hline \multirow[t]{3}{*}{ Low-dose } & Mean \pm SD & $21.7 \pm 7.6$ & $20.7 \pm 8.7$ & $13.5 \pm 9.7$ & $37.4 \pm 7.7$ & $49.0 \pm 8.8$ & $50.0 \pm 12.2$ & $44.3 \pm 8.0$ & $5.7 \pm 8.9$ \\
\hline & Min & 5.3 & -0.6 & -10.0 & 21.3 & 30.6 & 22.3 & 27.1 & -14.1 \\
\hline & Max & 43.2 & 49.3 & 33.7 & 58.1 & 66.9 & 84.9 & 60.9 & 28.6 \\
\hline \multirow[t]{3}{*}{ Micro-dose } & Mean \pm SD & $22.5 \pm 7.8$ & $21.0 \pm 9.8$ & $14.4 \pm 9.5$ & $37.5 \pm 8.3$ & $48.6 \pm 8.2$ & $50.7 \pm 10.8$ & $43.6 \pm 6.9$ & $7.1 \pm 8.1$ \\
\hline & Min & 6.8 & 5.5 & -5.9 & 16.5 & 31.3 & 23.9 & 30.7 & -11.4 \\
\hline & Max & 44.8 & 52.6 & 34.5 & 54.4 & 71.3 & 72.8 & 60.7 & 27.4 \\
\hline
\end{tabular}

Table 4 Intraclass correlation coefficients (ICC) with a 95\% confidence interval $(\mathrm{CI})$ for clinical parameters coming from 3D reconstructions performed with both low-dose and micro-dose

\begin{tabular}{lll}
\hline & $\begin{array}{l}\text { ICC }(95 \% \text { CI }) \\
\text { Low-dose }\end{array}$ & $\begin{array}{l}\text { ICC }(95 \% \text { CI }) \\
\text { Micro-dose }\end{array}$ \\
\hline Cobb angle & $0.88(0.74-0.94)$ & $0.90(0.79-0.95)$ \\
T1-T12 kyphosis & $0.84(0.66-0.92)$ & $0.73(0.48-0.87)$ \\
T4-T12 kyphosis & $0.79(0.57-0.90)$ & $0.92(0.84-0.97)$ \\
L1-L5 lordosis & $0.80(0.60-0.91)$ & $0.90(0.78-0.95)$ \\
L1-S1 lordosis & $0.81(0.62-0.91)$ & $0.77(0.52-0.89)$ \\
Pelvic incidence & $0.91(0.81-0.96)$ & $0.92(0.82-0.96)$ \\
Sacral slope & $0.81(0.61-0.91)$ & $0.86(0.70-0.94)$ \\
Pelvic tilt & $0.99(0.97-0.99)$ & $0.95(0.90-0.98)$ \\
\hline
\end{tabular}

spine measurements of the conventional EOS low-dose and micro-dose protocols in children wearing a brace.

The use of the EOS micro-dose protocol resulted in a reduction of the radiation exposure by a factor of 7 for the PA view and 5 for the LAT view, when compared to the low-dose protocol. In this study, the 3D models reconstructed from the micro-dose images had a good to very good interobserver agreement for all clinical measurements (ICC $>0.73$ ). Reliability of 3D measurements was comparable between the low-dose and micro-dose measurements, with no statistically significant difference amongst the clinical parameters that were assessed. Although not significantly different, mean inter-rater 3D measurement of T1T12 kyphosis was $5.5^{\circ} \pm 5^{\circ}$ with micro-dose versus $3.5 \pm 3.6^{\circ}$ with low-dose $(p=0.12)$. This discrepancy can be explained by two factors. First, even though the children were wearing a brace, motions (swaying or breathing) between acquisition may have occurred and led to small motion artefacts, which could have resulted in measurement differences between the low-dose and micro-dose acquisitions, while maintaining an excellent agreement between two observers. Second, as was observed during the image quality assessment, the micro-dose protocol provided lower image quality, particularly on the upper thoracic spine. Even though the microdose images remained interpretable, it may be difficult to visualize some specific parts of thoracic vertebrae, which may have led to increased inter-rater variability in the 3D reconstructions particularly in this region. The same difficulty has already been observed in previous study by Rehm et al. with low-dose protocol [20]. It could be explained by the fact that the axial rotation of the vertebra resulted mainly from the adjustment of the pedicles and the posterior arch in the frontal view. These structures can be difficult to identify in the upper and middle thoracic spine, because of the lower diameter and the obtuse angle of the pedicles and the posterior arch in comparison to the lumbar spine. However, radiologists can still determine the Cobb angle and obtain clinical measurements with a good reliability in braced children. It should also be noted that the measurement error observed here was within the reported reproducibility for the sterEOS measurements with low-dose protocol [20], and was similar to results that have been reported previously in the literature using standard radiographic methods [21]. These differences were not likely to affect the diagnostic or prognostic evaluation of the patient and were, therefore, not

Table 5 Comparison of inter-rater measurements (in degrees) between low-dose and micro-dose 3D models

\begin{tabular}{|c|c|c|c|c|c|c|c|c|}
\hline & Cobb angle & $\begin{array}{l}\text { T1-T12 kypho- } \\
\text { sis }\end{array}$ & $\begin{array}{l}\text { T4-T12 kypho- } \\
\text { sis }\end{array}$ & L1-L5 lordosis & L1-S1 lordosis & Pelvic incidence & Sacral slope & Pelvic tilt \\
\hline $\begin{array}{l}\text { Low-dose } \\
\quad(\text { mean } \pm \mathrm{SD})\end{array}$ & $2.9 \pm 2.5$ & $3.5 \pm 3.6$ & $4.8 \pm 4.9$ & $3.9 \pm 4.1$ & $3.7 \pm 2.9$ & $3.1 \pm 2.7$ & $3.5 \pm 3.8$ & $1.1 \pm 1.1$ \\
\hline $\begin{array}{l}\text { Micro-dose } \\
\quad(\text { mean } \pm \mathrm{SD})\end{array}$ & $2.6 \pm 2.4$ & $5.5 \pm 5.0$ & $3.1 \pm 2.3$ & $2.7 \pm 2.9$ & $3.7 \pm 3.4$ & $3.8 \pm 3.9$ & $2.6 \pm 2.7$ & $1.6 \pm 2.2$ \\
\hline$p$ value & 0.53 & 0.12 & 0.14 & 0.26 & 0.62 & 0.16 & 0.51 & 0.18 \\
\hline
\end{tabular}


clinically significant. Pasha et al. have recently stressed that significant differences between the spinal and pelvic parameters suggest that the position during the EOS scan (wall and clavicle arm positions) should not be used interchangeably in AIS postural assessment [22].

As reported by Newton et al., the standard EOS low-dose images provided better image quality and should be used for initial diagnostic evaluation and preoperative assessment in AIS [14]. The micro-dose images would be best utilized for following curve progression after the initial diagnosis has been made, and for evaluating the in-brace spine curvature in AIS patients. The EOS micro-dose protocol enabled a reliable spine assessment with significant dose reduction in this population that is regularly exposed to ionizing radiation. Branchini et al. have highlighted that the shielding of testes and the exclusion of eyes from the EOS scan could allow to further reduce the dose [23]. The long-term impact of the decreased cumulative radiation exposure on the risk of developing cancer for these children would require prospective studies.

\section{Conclusions}

The use of the EOS micro-dose protocol resulted in a significant reduction of the delivered dose, by a factor of 5-7 compared to the conventional low-dose protocol, while maintaining an interpretable image quality and good interrater agreement of 3D spine measurements in braced children. The micro-dose protocol constitutes a very promising tool for the follow-up of children with adolescent idiopathic scoliosis.

Funding No funding was secured for this study.

\section{Compliance with ethical standards}

Conflict of interest The authors have no conflicts of interest to disclose. The authors have no financial relationships relevant to this article to disclose.

Ethical statement The local institutional review board approved this single center, non-interventional, prospective study.

\section{References}

1. Ilharreborde B, Sebag G, Skalli W, Mazda K (2013) Adolescent idiopathic scoliosis treated with posteromedial translation: radiologic evaluation with a 3D low-dose system. Eur Spine J 22:2382-2391

2. Hong J-Y, Suh S-W, Easwar TR et al (2011) Evaluation of the three-dimensional deformities in scoliosis surgery with computed tomography: efficacy and relationship with clinical outcomes. Spine 36:E1259-E1265
3. Courvoisier A, Drevelle X, Dubousset J, Skalli W (2013) Transverse plane 3D analysis of mild scoliosis. Eur Spine J 22:2427-2432

4. Nault M-L, Mac-Thiong J-M, Roy-Beaudry M et al (2014) Three-dimensional spinal morphology can differentiate between progressive and non-progressive patients with adolescent idiopathic scoliosis at the initial presentation. Spine 39(10):E601

5. Blumer SL, Dinan D, Grissom LE (2014) Benefits and unexpected artifacts of biplanar digital slot-scanning imaging in children. Pediatr Radiol 44:871-882

6. Pearce MS, Salotti JA, Little MP et al (2012) Radiation exposure from CT scans in childhood and subsequent risk of leukaemia and brain tumours: a retrospective cohort study. Lancet 380:499-505

7. Mathews JD, Forsythe AV, Brady $Z$ et al (2013) Cancer risk in 680000 people exposed to computed tomography scans in childhood or adolescence: data linkage study of 11 million Australians. BMJ 346:f2360

8. Bone CM, Hsieh GH (2000) The risk of carcinogenesis from radiographs to pediatric orthopaedic patients. J Pediatr Orthop $20: 251-254$

9. Levy AR, Goldberg MS, Mayo NE et al (1996) Reducing the lifetime risk of cancer from spinal radiographs among people with adolescent idiopathic scoliosis. Spine 21:1540-1547 (discussion 1548)

10. Law M, Ma W-K, Lau D et al (2016) Cumulative radiation exposure and associated cancer risk estimates for scoliosis patients: impact of repetitive full spine radiography. Eur $\mathrm{J}$ Radiol 85:625-628

11. Ronckers CM, Land CE, Miller JS et al (2010) Cancer mortality among women frequently exposed to radiographic examinations for spinal disorders. Radiat Res 174:83-90

12. Sodhi KS, Krishna S, Saxena AK et al (2015) Clinical application of "justification" and "optimization" principle of ALARA in pediatric CT imaging: "how many children can be protected from unnecessary radiation?' Eur J Radiol 84(9):1752-1757

13. Ilharreborde B, Ferrero E, Alison M, Mazda K (2016) EOS microdose protocol for the radiological follow-up of adolescent idiopathic scoliosis. Eur Spine J 25:526-531

14. Newton PO, Khandwala Y, Bartley CE et al (2016) New EOS imaging protocol allows a substantial reduction in radiation exposure for scoliosis patients. Spine Deform 4:138-144

15. Dubousset J, Ilharreborde B, Le Huec J-C (2014) Use of EOS imaging for the assessment of scoliosis deformities: application to postoperative 3D quantitative analysis of the trunk. Eur Spine J 23(4):397-405

16. European Union. European Commission; Directorate-General XII-Science R and D (1996) European guidelines on quality criteria for diagnostic radiographic images in paediatrics. Office for Official Publications of the European Communities

17. Deschênes S, Charron G, Beaudoin G et al (2010) Diagnostic imaging of spinal deformities: reducing patients radiation dose with a new slot-scanning X-ray imager. Spine 35:989-994

18. Presciutti SM, Karukanda T, Lee M (2014) Management decisions for adolescent idiopathic scoliosis significantly affect patient radiation exposure. Spine J 14:1984-1990

19. Law M, Ma W-K, Chan E et al (2017) Evaluation of cumulative effective dose and cancer risk from repetitive full spine imaging using EOS system: impact to adolescent patients of different populations. Eur J Radiol 96:1-5

20. Rehm J, Germann T, Akbar M et al (2017) 3D-modeling of the spine using EOS imaging system: inter-reader reproducibility and reliability. PLoS ONE 12:e0171258

21. Carreau JH, Bastrom T, Petcharaporn M et al (2014) Computer-generated, three-dimensional spine model from biplanar 
radiographs: a validity study in idiopathic scoliosis curves greater than 50 degrees. Spine Deform 2:81-88

22. Pasha S, Capraro A, Cahill PJ et al (2016) Bi-planar spinal stereoradiography of adolescent idiopathic scoliosis: considerations in 3D alignment and functional balance. Eur Spine J 25:3234-3241
23. Branchini M, Del Vecchio A, Gigliotti CR et al (2017) Organ doses and lifetime attributable risk evaluations for scoliosis examinations of adolescent patients with the EOS imaging system. Radiol Med. https://doi.org/10.1007/s11547-017-0828 